\title{
Tuneable coupling of surface plasmon-polaritons and Mie plasmons on a planar surface of nanoporous metal
}

\author{
T.V. Teperik ${ }^{* 1}$, V.V. Popov ${ }^{1}$, F.J. García de Abajo ${ }^{2}$, and J.J. Baumberg ${ }^{3}$ \\ 1 Institute of Radio Engineering and Electronics (Saratov Division), Russian Academy of Sciences, \\ Saratov 410019, Russia \\ 2 Centro Mixto CSIC-UPV/EHU and Donostia International Physics Center (DIPC), Aptdo. 1072, \\ 20080 San Sebastian, Spain \\ 3 Department of Physics and Astronomy, University of Southampton, Southampton, SO17 1BJ, \\ United Kingdom
}

Received 8 June 2005, accepted 18 June 2005

Published online 10 November 2005

PACS 61.46.+w, 73.20.Mf, 78.20.Bh

We have studied optical plasmonic properties of metal surfaces with a periodic lattice of voids buried in metal just beneath the surface. We have calculated reflection spectra of such surfaces in the framework of a self-consistent electromagnetic multiple-scattering layer-KKR approach. We investigated two types of plasmon excitations: surface plasmon-polaritons propagating at the planar surface of metal and Mie plasmons localized in voids. The coupling between these two types of plasma oscillations can be modified by tuning the structure parameters (e.g., void diameter and/or the period of the void lattice) or by changing the angle of incidence of incoming light.

() 2005 WILEY-VCH Verlag GmbH \& Co. KGaA, Weinheim

\section{Introduction}

Currently strong efforts are focussed on investigating the optical properties of metals with periodically arranged nanopores. Interest in such structures is based on considerable advances in the template-assisted assembly of microporous and nanoporous metal structures [1-3]. Remarkable effects caused by excitation of plasmons in nanopores have been predicted theoretically for nanoporous metal films, such as extraordinary transmission [4] and absorption [5]. In [1] strong resonant dips in the reflectivity spectra of light have been observed from nanoporous metal surfaces formed by periodical arrangements of closepacked spherical segment voids (nanocups). All these effects are caused by the excitation of Mie plasmons in the voids (void plasmons). However, apart from the Mie plasmons localized in spherical cavities inside the metal, propagating surface plasmon-polaritons can be also excited, in principle, at the planar surface of nanoporous metal if the period of the void lattice beneath the surface is comparable with the surface plasmon-polariton wavelength. The lattice of voids buried beneath the planar surface of metal plays a two-fold role in this case: (i) the periodic lattice of voids forms a coupling element, which couples incoming light to surface plasmon-polaritons and (ii) localized Mie plasmons are excited in the spherical voids.

In this paper, we study the optical plasmonic properties of a planar metal surface with a hexagonal two-dimensional (2D) lattice of spherical nanovoids buried inside metal at some distance from the surface. We have calculated the reflectivity spectra of such a nanoporous metal surface in the framework of a self-consistent electromagnetic multiple-scattering layer-Korringa-Kohn-Rostoker (KKR) approach [4-

\footnotetext{
* Corresponding author: e-mail: teperik@soire.renet.ru, Phone: 7 (8452) 272401, Fax: 7 (8452) 272401
} 
6]. We investigate two types of plasmon excitations: surface plasmon-polaritons excited at the planar surface of metal and Mie plasmons localized in voids. The special attention is given to coupling effects between these two types of plasma oscillations.

\section{Results and discussion}

Let us consider light incident at an angle $\theta$ to the surface normal (Fig. 1) of a planar surface of metal that contains a $2 \mathrm{D}$ lattice of voids just beneath the surface. The lattice has primitive vectors $\mathbf{a}$ and $\mathbf{b}$, where $\mathbf{a}=\mathbf{b}$ and $\mathbf{a} \cdot \mathbf{b}=|\mathbf{a}|^{2} \cos 60^{\circ}$. The plane of incidence of external light is defined by the azimuthal angle $\varphi$ measured with respect to the $x$-axis (Fig. 1). To calculate the light reflected from such a porous surface we use a rigorous solution of Maxwell's equations based upon a multiple-scattering layer-KorringaKohn-Rostoker (KKR) approach that makes use of a re-expansion of the plane-wave representation of the electromagnetic field in terms of spherical harmonics [4-6]. We describe the dielectric response of the metal to a light electric field $\mathbf{E} \exp (-i \omega t)$ in the local Drude model as

$$
\varepsilon(\omega)=1-\frac{\omega_{p}^{2}}{\omega\left(\omega+i v_{e}\right)},
$$

where $\omega_{p}$ is the bulk plasmon frequency and $v_{e}$ is a phenomenological bulk electron relaxation rate. For our calculations we choose the parameters $\omega_{p}=7.9 \mathrm{eV}$ and $v_{e}=90 \mathrm{meV}$, which are characteristic for gold [7].
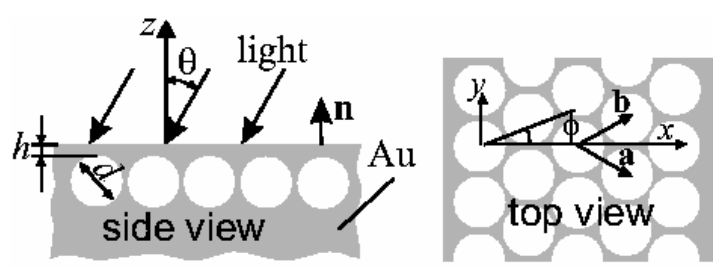

Fig. 1 Nanoporous metal surface with a 2D hexagonal lattice of spherical voids.

In Fig. 2 calculated reflection spectra of $s$ - and $p$-polarized light as a function of photon energy $\hbar \omega$ and in-plane light wavevector $k_{\|}$are shown for a planar gold surface with a single buried 2D hexagonal lattice $(|\mathbf{a}|=705 \mathrm{~nm})$ of voids of diameter $\mathrm{d}=630 \mathrm{~nm}$. Plane of incidence of incoming light is defined by the azimuthal angle $\phi=15^{\circ}$. The lattice of voids is buried beneath the planar surface of metal at distance $h$ of the order of the skin depth (that is about $25 \mathrm{~nm}$ for gold). One can see a series of dips in the reflectivity spectra. The stronger and almost dispersionless resonance is associated with excitation of Mie plasmons in the voids [5]. The frequency of the Mie-plasmon resonance is close to the frequency of the Mie-plasmon mode with orbital quantum number $l=2$ of a single void in bulk gold (marked by horizontal dashed line in Fig. 2. The other (dispersive) resonances in the reflectivity spectra are related to the excitation of surface plasmon-polaritons on the planar metal surface. The frequencies of these resonances are close to the frequencies of surface plasmon-polariton modes, which are estimated in the empty lattice approximation (marked by dashed curves in Fig. 2):

$$
q_{p q}^{2}=\frac{(\omega / c)^{2} \varepsilon(\omega)}{(1+\varepsilon(\omega))},
$$

where $\mathbf{q}_{p q}=\mathbf{k}_{\|}+\mathbf{g}_{p q}$ are the surface plasmon-polariton wavevectors, $\mathbf{g}_{p q}=p \mathbf{A}+q \mathbf{B}$ are the in-plane reciprocal lattice vectors, $\mathbf{A}=2 \pi(\mathbf{b} \times \mathbf{n}) /|\mathbf{a} \times \mathbf{b}|$ and $\mathbf{B}=2 \pi(\mathbf{n} \times \mathbf{b}) /|\mathbf{a} \times \mathbf{b}|$ are the primitive vectors of the reciprocal 2D lattice, $p$ and $q$ are integers, and $k_{\|}=(\omega \sin \theta) / c$ is the in-plane component of the incident light wavevector, which is equal to zero in the case of normal incidence. 
In general, at arbitrary azimutal angle there are six surface plasmon-polariton resonances exhibited in the reflectivity spectra of obliquely incident light $(\theta \neq 0)$. This can be understood from Fig. 3 , where the first Brillouin zone and wavevectors of surface plasmon-polaritons belonging to the lowest energy subband $(|q| \leq 1,|p| \leq 1)$ are shown schematically. Thus, the surface plasmon-polariton resonance is 6-fold degenerate for normally incident light due to the hexagonal symmetry of the void lattice.
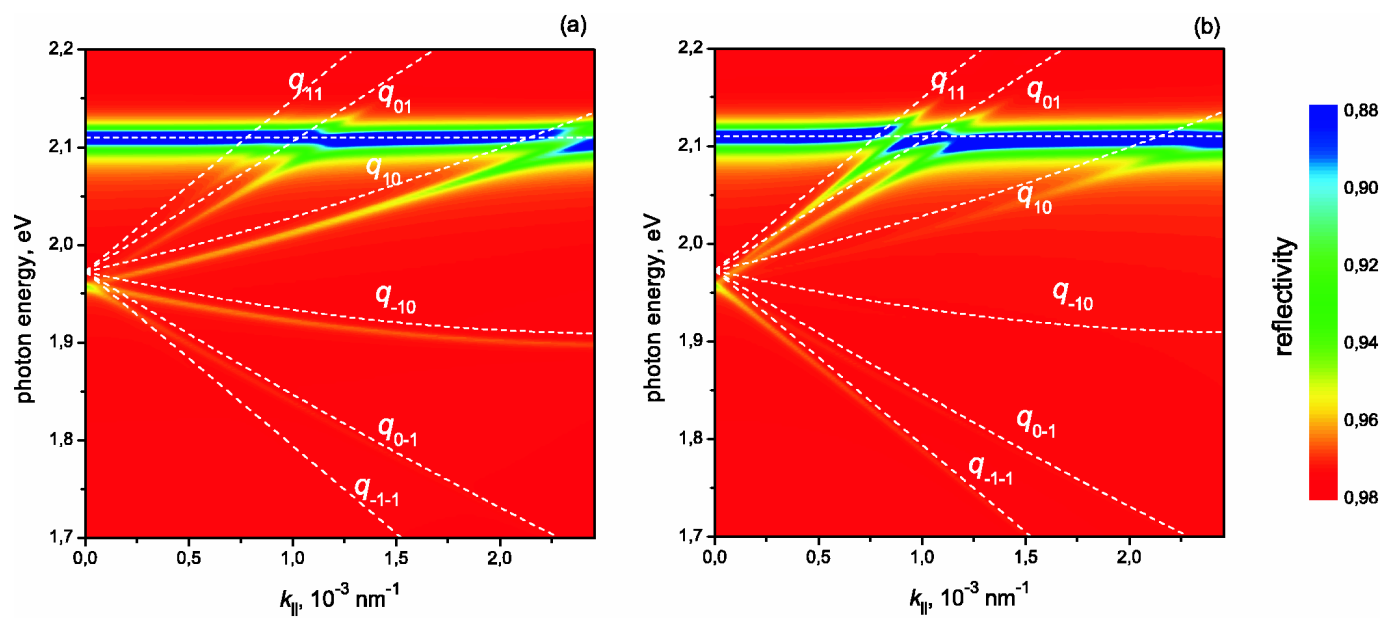

Fig. 2 Reflectivity spectra for (a) $s$-polarised and (b) $p$-polarised light incident in the plane of incidence with azimuthal angle $\phi=15^{\circ}$ onto the planar gold surface with an hexagonal lattice of spherical voids of diameter $\mathrm{d}=630 \mathrm{~nm}$ as a function of photon energy $\hbar \omega$ and in-plane light wavevector. The in-plane wavevector $k_{\|}$is related to the angle of incidence of incomimg light by $k_{\|}=\omega \sin \theta / c$. The distance from the planar surface of metal to the tops of voids, $h$, is equal to $25 \mathrm{~nm}$ and the lattice constant is $|\mathbf{a}|=|\mathbf{b}|=705 \mathrm{~nm}$. Dashed lines labelled with $q_{\mathrm{p}, \mathrm{q}}$ indicate the energies of the surface plasmon-polaritons estimated in the empty lattice approximation. The horizontal dashed line in each plot marks the energy of the second Mie-plasmon mode $(l=2)$ of a single void in bulk gold.

Note that different surface plasmon-polaritons are excited by $p$-polarized or $s$-polarized light with different efficiency even at a small angle of incidence, when the in-plane components of electric field for the $p$-polarized and $s$-polarized light have nearly equal amplitudes of the in-plane electric field. In the case of a small angle of incidence one has $\left|\mathbf{k}_{\|}\right|<<\left|\mathbf{g}_{p q}\right|$ and, hence, the direction of propagation of different surface plasmon-polariton modes are nearly parallel to the reciprocal lattice vectors $\mathbf{g}_{p q}$ (Fig. 3). Therefore, for a certain azimuthal angle $\phi=15^{\circ}$ as that in Fig. 2, surface plasmon-polaritons with $(p, q)=(1,1)$ and $(p, q)=(-1,-1)$ are effectively excited by $p$-polarized light (see Fig. 2$)$ because the in-plane electric field component of $p$-polarized light $\mathbf{E}_{\|}^{(p)}$ is nearly parallel to the wavevectors of these particular surface plasmon-polaritons, which nearly coincide with vectors $\mathbf{A}+\mathbf{B}$ and $-(\mathbf{A}+\mathbf{B})$, respectively. Due to the same reason $s$-polarized light effectively excites surface plasmon-polaritons with $(p, q)=(1,0)$ and $(p, q)=(-1,0)$, which have the wavevectors nearly parallel to vectors $\mathbf{A}$ and $-\mathbf{A}$, respectively. Surface plasmon-polaritons with $(p, q)=(0,-1)$ and $(p, q)=(0,1)$ are excited by $p$-polarized and $s$-polarized light with nearly the same moderate efficiency (see Fig. 2) since the in-plane electric-field components of either $p$-polarized or $s$-polarized light comprises an angle about $45^{\circ}$ with wavevectors $\mathbf{q}_{0,-1}$ and $\mathbf{q}_{0,1}$.

One can see in Fig. 2 that Mie-plasmon resonance and surface plasmon-polariton resonance exhibit clear anticrossing when Mie-plasmon resonance passes by a surface plasmon-polariton resonance with variation of the in-plane wavevector $k_{\|}$. This proves that these two types of plasmon resonances effec- 
tively interact with each other. Note, that in general, Mie plasmons excited in voids produce a stronger resonance dip in comparison with that of surface plasmon-polariton resonance. The reason for this is that Mie plasmons in voids are radiative excitations [8] and, hence, they can couple to light effectively, while surface plasmons are non-radiative excitations and can be coupled to light only via a coupling element, which is the lattice of voids itself in the case under consideration. Notice, however, that in the anticrossing regime Mie-plasmon resonance and surface plasmon-polariton resonance become comparable in amplitude. This is because in the interaction region these two types of plasmon resonance effectively mix their oscillator strength, so that the stronger Mie-plasmon resonance supplies a weaker surface plasmonpolariton resonance with additional oscillator strength. As a result, the strength of the surface plasmonpolariton resonance increases in the interaction region.

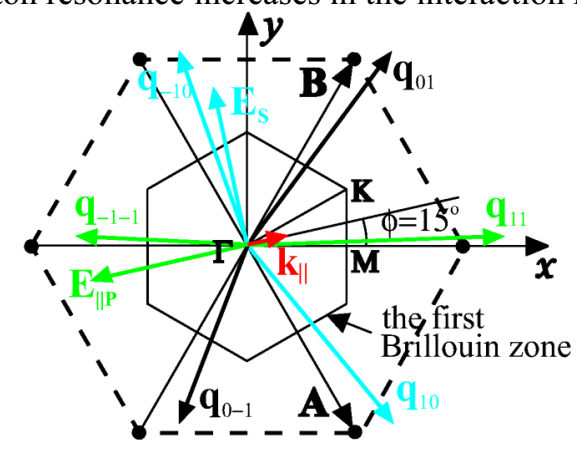

Fig. 3 The first Brillouin zone and the wavevectors $\boldsymbol{q}_{\mathrm{p}, \mathrm{q}}$ of surface plasmon-polaritons in the lowest frequency subband.

In conclusion, the coupling between surface plasmon-polaritons and Mie plasmons can be effectively modified by tuning the structure parameters (e.g., void diameter and/or the period of the void lattice) or by changing the angle of incidence of incoming light and its plane of incidence orientation. This effect is of broad general interest in the context of future optical applications such as spectroscopy of surface epilayers and absorbates in optoelectronics and biophysics.

Acknowledgements This work was supported by the Russian Academy of Sciences, Russian Foundation for Basic Research (Grant 05-02-17513) and UK EPSRC EP/C511786/1. T.V.T. acknowledges the support from the National Foundation for Promotion of Science, and also thanks the Donostia International Physics Center in San Sebastian, Spain, for hospitality during the preparation of this work. F.J.G.A. acknowledges support from the Spanish MECD (contract No. FIS2004-06490-C03-02) and from the EU (Metamorphose NoE NMP3-CT-2004-500252).

\section{References}

[1] S. Coyle, M.C. Netti, J.J. Baumberg et al., Phys. Rev. Lett. 87, 176801 (2001).

[2] O. D. Velev, P. M. Tessier, A. M. Lenhoff et al., Nature 401, 548 (1999).

[3] J. E. G. J. Wijnhoven, S. J. M. Zevenhuizen, M. A. Hendriks et al., Adv. Mater. 12, 888 (2000).

[4] N. Stefanou, A. Modinos, and V. Yannopapas, Solid State Commun. 69, 118 (2001).

[5] T.V. Teperik, V.V. Popov, and F.J. García de Abajo, Phys. Rev. B 71, 085408 (2005).

[6] N. Stefanou, V. Yannopapas, and A. Modinos, Comput. Phys. Commun. 113, 49 (1998).

[7] M. Kreiter, S. Mittler, W. Knoll, and J. R. Sambles, Phys. Rev. B 65, 125415 (2002).

[8] T.V. Teperik, V.V. Popov, and F.J. García de Abajo, Phys. Rev. B 69, 155402 (2004). 